\title{
So-Cheong-Ryoung-Tang Attenuates Pulmonary Inflammation Induced by Cigarette Smoke in Bronchial Epithelial Cells and Experimental Mice
}

\author{
Na-Rae Shin', Chul Kim², Chang-Seob Seo ${ }^{2}$, Je-Won Ko', Young-Kwon Cho ${ }^{3}$, \\ Jong-Choon Kim ${ }^{1}$, Joong-Sun Kim ${ }^{2 *}$ and In-Sik Shin ${ }^{1 *}$ \\ ${ }^{1}$ BK21 Plus Project Team, College of Veterinary Medicine, Chonnam National University, Gwangju, South Korea, \\ ${ }^{2}$ Herbal Medicine Research Division, Korea Institute of Oriental Medicine, Daejeon, South Korea, ${ }^{3}$ College of Health \\ Sciences, Cheongju University, Cheongju, South Korea
}

OPEN ACCESS

Edited by:

Min Ye,

Peking University, China

Reviewed by:

Ki-Tae Ha,

Pusan National University,

South Korea

Shuai Ji,

Xuzhou Medical University, China

*Correspondence:

Joong-Sun Kim

centraline@kiom.re.kr

In-Sik Shin

dvmmk79@gmail.com

Specialty section:

This article was submitted to

Ethnopharmacology,

a section of the journal

Frontiers in Pharmacology

Received: 21 June 2018 Accepted: 03 September 2018 Published: 21 September 2018

Citation:

Shin N-R, Kim C, Seo C-S, Ko J-W,

Cho Y-K, Kim J-C, Kim J-S and Shin I-S (2018)

So-Cheong-Ryoung-Tang Attenuates

Pulmonary Inflammation Induced by

Cigarette Smoke in Bronchial

Epithelial Cells and Experimental

Mice. Front. Pharmacol. 9:1064.

doi: 10.3389/fphar.2018.01064
So-Cheong-Ryoung-Tang is a traditionally used herbal formula for the treatment of pulmonary diseases in China, Korea, and Japan. We investigated the protective effects of So-Cheong-Ryong-Tang water extract (SCWE) in cigarette smoke concentrate (CSC) stimulated human airway epithelial cell line $\mathrm{NCl}-\mathrm{H} 292$ and mice exposed cigarette smoke (CS) and lipopolysaccharide (LPS). In the CSC-stimulated NCl-H292 cells, SCWE inhibited proinflammatory cytokines in a concentration-dependent manner, as evidenced by a reduction in their mRNA levels. Also, SCWE significant reduced inducible nitric oxide synthase (iNOS) expression and nuclear factor kappa B (NF-кB) phosphorylation in CSC-stimulated cells. The mice were exposed to CS for $1 \mathrm{~h}$ per day (a total of eight cigarettes per day) for 7 days and received LPS intranasally on day 5 . The mice were administered a dose of SCWE (100 and $200 \mathrm{mg} / \mathrm{kg}) 1 \mathrm{~h}$ before CS exposure. In in vivo, SCWE decreased the inflammatory cell count and reduced the expression of the proinflammatory cytokines in the broncho-alveolar lavage fluid (BALF) compared with CS and LPS exposed mice. SCWE attenuated inflammatory cell infiltration in airway induced by CS and LPS exposure, and this decrease was accompanied by a reduction in the expression levels of iNOS and MMP-9 in lung tissue. The extract also inhibited

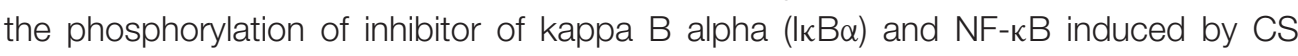
and LPS exposure in lung tissue. These results suggest that SCWE may effectively inhibit airway inflammatory responses induced by CS and LPS exposure via the NFкB pathway. Therefore, SCWE may be a potential treatment for airway inflammatory diseases, such as chronic obstructive pulmonary disease (COPD).

Keywords: So-Cheong-Ryong-Tang, cigarette smoke, iNOS, MMP-9, NF-кB

\section{INTRODUCTION}

Chronic obstructive pulmonary disease (COPD) is a chronic inflammatory disease of the airway. The pathology of COPD is characterized by neutrophilic airway inflammation, airway remodeling, and pulmonary emphysema in response to cigarette smoke (CS) exposure (Dong et al., 2015; Jung et al., 2016a). CS is a complex mixture of oxidant radicals and various chemical compounds, 
including reactive aldehydes and semiquinones. CS induces harmful effects on human health and is widely recognized as a primary risk factor for the progression of COPD (Ge et al., 2016). CS exposure leads to airway inflammation, enhanced levels of proinflammatory cytokines and chemokines, and airspace enlargement (Jung et al., 2016b). CS has been shown to increase the infiltration of inflammatory cells such as macrophages and neutrophils that are directly involved in the release of matrix metalloproteinases (MMPs; Lee et al., 2013). MMPs are involved in the development of COPD via modulation of cytokine and chemokine production, extracellular matrix turnover, and tissue remodeling (Cane et al., 2016). MMP-9 has been shown to destroy the normal airway structure and induce airway inflammation. In a clinical trial, MMP-9 was found in the sputum and lavage samples of patients with COPD, and its expression was associated with activation of nuclear factor kappa $B(N F-\kappa B)$, resulting in inflammatory responses (Pang et al., 2015).

Nuclear factor kappa B is a family of transcription factors involved in the regulation of proinflammatory and immune regulatory pathways (Jones et al., 2016). Activated NF- $\kappa$ B induces the expression of many inflammatory mediators, including inducible nitric oxide synthase (iNOS; Kwon et al., 2016). iNOS has been implicated in the pathophysiology of pulmonary disease and is upregulated by various proinflammatory signals. The resulting increase in $\mathrm{NO}$ concentration is a part of the immediate immune defense reaction (Hesslinger et al., 2009). Previous studies have demonstrated that iNOS is an important mediator in COPD patients and in the CS-induced mouse model (Hesslingere et al., 2008; Jiang et al., 2015).

So-Cheong-Ryoung-Tang is a traditional oriental medicine and is known as Xiao-Qing-Long-Tang in China and Sho-SeiryuTo in Japan (Park et al., 2015). It is composed a mixture of eight herbal preparations (Herba Ephedrae, Radix Paeoniae, Fructus Schisandrae, Tuber Pinelliae, Asiasari Radix, Rhizoma Crudus Zingiberis, Ramulus Cinnamomi, and Radix et Rhizoma Glycyrrhizae). We searched electronic databases for relevant studies published before May 2018 in the Oriental Medicine Advanced Searching Integrated System (OASIS). According to the OASIS, this mixture has long been used to treat common cold, bronchitis, and bronchial asthma in the traditional oriental clinic. It is an herbal mixture used to treat various diseases such as allergic rhinitis and asthma (Yang et al., 2009; Wang et al., 2012; Kim et al., 2014; Ku et al., 2016). It was recently shown that So-Cheong-Ryoung-Tang effectively decreased the production of cytokines and chemokines on airway remodeling in allergic asthma model and attenuated allergic effects in a rat model of ovalbumin-induced rhinitis via the phosphatidylinositol 3 kinase (PI3K)/Akt signaling pathway (Kim et al., 2014; Park et al., 2015). However, there is no study available on the effect of So-Cheong-Ryong-Tang on airway inflammation induced by CS and lipopolysaccharide (LPS) exposure.

Therefore, we explored the anti-inflammatory effect of So-Cheong-Ryong-Tang water extract (SCWE) on airway inflammation induced by CS and LPS exposure. To confirm the possible underlying mechanism of SCWE, we investigated the expression and production of inflammatory mediators in a model of CS and LPS induced airway inflammation.

\section{MATERIALS AND METHODS}

\section{Plant Materials}

So-Cheong-Ryong-Tang water extract consisted of eight herbal medicines, and raw materials were purchased from Omniherb (Yeongcheon, Korea) and HMAX (Jecheon, Korea) in February 2008. The identification of eight raw materials was performed by Prof. Je-Hyun Lee, College of Oriental Medicine, Dongguk University (Gyeongju, Korea). A voucher specimen (2008KE13-1 KE13-8) was deposited at the K-herb Research Center, Korea Institute of Oriental Medicine.

\section{Chemicals and Materials}

Coumarin (purity $\geq 99.0 \%$ ) and cinnamic acid (purity $\geq 98.0 \%$ ) were purchased from Sigma-Aldrich (St. Louis, MO, United States). Albiflorin (purity $\geq 99.0 \%$ ), paeoniflorin (purity $\geq 99.0 \%$ ), cinnamaldehyde (purity $\geq 98.0 \%$ ), glycyrrhizin (purity $\geq 99.0 \%$ ), and schizandrin (purity $\geq 99.0 \%$ ) were purchased from Wako (Osaka, Japan). Liquiritin (purity $\geq 98.0 \%$ ) was purchased from NPC BioTechnology Inc. (Daejeon, Korea). High-performance liquid chromatography (HPLC)-grade methanol, acetonitrile, and water were purchased from J.T. Baker (Phillipsburg, NJ, United States). Glacial acetic acid (reagent grade) was purchased from Junsei (Tokyo, Japan).

\section{Preparation of SCWE}

So-Cheong-Ryong-Tang water extract decoction was prepared from a mixture of eight medicinal herbs in a ratio as shown in Supplementary Table S1 (total weight $=3.5 \mathrm{~kg}$, about 93.3 times the composition of a single dose). The mixture was extracted in $35.0 \mathrm{~L}$ of distilled water at $100^{\circ} \mathrm{C}$ for $2 \mathrm{~h}$ using an electric extractor (COSMOS-660; Kyungseo Machine Co., Incheon, Korea). The solution was filtered using a standard sieve (No. 270, $53 \mu \mathrm{m}$; Chung Gye Sang Gong Sa, Seoul, Korea), evaporated to dryness at $50^{\circ} \mathrm{C}$ under vacuum using an evaporator Eyela N-11 (Tokyo, Japan), and then freeze-dried using a vacuum control freeze dryer (PVTFD10RS, IlShinBioBase, Yangju, Korea) to give a powder of extract sample. As a result, the amount of lyophilized SCWE powder was obtained $760.6 \mathrm{~g}$ (yield: $21.7 \%$ ).

\section{HPLC Analysis of SCWE Decoction}

The quantitative analysis of the eight biomarker compounds in SCWE decoction was performed using a Shimadzu Prominence LC-20A system (Kyoto, Japan) equipped with LC-20AT pumps, DGU-20A3 online degasser, CTO-20A column oven, SIL-20AC auto sample injector, and SPD-M20A photodiode array (PDA) detector. All chromatographic data were recorded and processed by LCsolutions software (Version 1.24 SP1). The separation of the eight analytes was achieved on a Phenomenx Gemini C18 column $(250 \mathrm{~mm} \times 4.6 \mathrm{~mm}, 5 \mu \mathrm{m}$, Torrance, CA, United States), and column temperature was maintained at $40^{\circ} \mathrm{C}$. The mobile phase consisted of $1.0 \%(\mathrm{v} / \mathrm{v})$ aqueous acetic acid (A) and 1.0\% (v/v) acetic acid in acetonitrile (B), and gradient conditions were as follows: $15-65 \% \mathrm{~B}$ (0-35 $\mathrm{min})$, $65-100 \%$ B (35-45 $\mathrm{min}), 100 \% \mathrm{~B}$ (45-50 $\mathrm{min}$ ), and $100-15 \%$ $\mathrm{B}(50-55 \mathrm{~min})$. The flowrate was $1.0 \mathrm{~mL} / \mathrm{min}$, and the injection 
volume was $10 \mu \mathrm{L}$. For quantitative analysis, lyophilized $200 \mathrm{mg}$ of SCWE extract was dissolved in $20 \mathrm{~mL}$ of distilled water and extracted at room temperature for 20 min using an ultrasonicator (Branson 8510, Danbury, CT, United States) The solution was then filtered through a $0.2 \mu \mathrm{m}$ membrane filter (PALL Life Sciences, Ann Arbor, MI, United States) before the HPLC injection.

\section{Cell Culture and Cell Viability}

A mucoepidermoid pulmonary carcinoma cell, NCI-H292, was maintained in RPMI 1640 supplemented with 10\% heatinactivated fetal bovine serum (FBS) and antibiotics at $37^{\circ} \mathrm{C}$ in a $5 \% \mathrm{CO}_{2}$ incubator and $95 \%$ air. The cell viability in response to SCWE was measured using a 3-(4,5-dimethylthiazol-2-yl)-2,5diphenyltetrazolium bromide (MTT) assay. The NCI-H292 cells were cultured in 96-well plates at a density of $3 \times 10^{4}$ cells/well. The SCWE was added to each individual well at concentrations of $1.25,2.5,5$, and $10 \mu \mathrm{g} / \mathrm{mL}$, followed by incubation for $24 \mathrm{~h}$. MTT solution $(10 \mu \mathrm{L})$ was added to each well, and the cells were incubated for $4 \mathrm{~h}$ at $37^{\circ} \mathrm{C}$. After incubation, $100 \mu \mathrm{L}$ of dimethyl sulfoxide (DMSO) was added to each well to solubilize the produced formazan. The optical density was measured at $570 \mathrm{~nm}$ using a microplate reader (Molecular Devices Ins, CA, United States).

\section{Reverse Transcriptase-Polymerase Chain Reaction (RT-PCR)}

The cells were seeded on $60 \mathrm{~mm}$ dish at a density of $1 \times 106$ cells/well, treated with a non-toxic concentration of SCWE and incubated in the presence of cigarette smoke concentrate (CSC; $20 \mu \mathrm{g} / \mathrm{mL}$ ). To investigate the effect of melatonin on TNF- $\alpha$, IL- 6 and IL- $1 \beta$ expression, the cells were treated with $\operatorname{SCWE}(1.25,2.5,5$, and $10 \mu \mathrm{g} / \mathrm{mL})$. The cells were harvested $24 \mathrm{~h}$ after SCWE treatment. The total RNA was isolated using TRIZOL ${ }^{\mathrm{TM}}$ Reagent (Invitrogen, Carlsbad, CA, United States). For RT-PCR, single-strand cDNA was synthesized from $1 \mu \mathrm{g}$ of total RNA. The polymerase chain reactions were performed using specific forward and reverse primers (TNF- $\alpha$, forward, $5^{\prime}$-CAAAGTAGACCTGCCCAGAC$3^{\prime}$, reverse, $5^{\prime}$-GACCTCTCTCTA ATCAGCCC-3'; IL-6, forward, $5^{\prime}$-ATGCAATAACCACCCCTGAC- $3^{\prime}$ and reverse, $5^{\prime}$ - ATCTGA GGTGCCCATGCTAC-3'; IL-1 $\beta$, forward, 5'-AGCCAGGACA GTCAGCTCTC-3' and reverse, 5'-ACTTCTTGCCCCCTTT GAAT-3'; GAPDH, forward, 5'-CAAAAG GGTCATCTCTG3', reverse, 5'-CCTGCTTCACCACCTTCTTG-3'). The PCR products were fractionated via $1.5 \%$ agarose gels electrophoresis and stained with $5 \mu \mathrm{g} / \mathrm{mL}$ ethidium bromide.

\section{Animals}

Specific pathogen-free male C57BL/6N mice (20-25 g, 6-8 weeks old) were purchased from the Samtako Co. (Osan, Korea). They were housed in groups of nine under standard conditions (temperature $22 \pm 2^{\circ} \mathrm{C}$, humidity $55 \pm 5 \%$, 12-h-light/dark cycle) with food and water available ad libitum. All experimental procedures were approved by the Institutional Animal Care and Use Committee of the Chonnam National University.

\section{Induction of CS and LPS in C57BL/6 Mice and Drug Administration}

The CS was generated from a 3R4F research cigarette (Kentucky reference cigarette, University of Kentucky, United States), containing $11.0 \mathrm{mg}$ of total particulate matter, $9.4 \mathrm{mg}$ of tar, and $0.76 \mathrm{mg}$ of nicotine per cigarette. Exposure of CS (one puff/min, $35 \mathrm{~mL}$ puff volume over $2 \mathrm{~s}$, every $60 \mathrm{~s}$, eight cigarettes per day) was conducted using a CS generator (Daehan Biolink, Seoul, Korea). The mice received $1 \mathrm{~h} C S$ exposure in the exposure chamber $(28 \mathrm{~cm} \times 28 \mathrm{~cm} \times 28 \mathrm{~cm})$ for 7 days. LPS $(10 \mu \mathrm{g}$ dissolved in $30 \mu \mathrm{L}$ distilled water) was intranasally instilled under anesthesia on day 5. SCWE was obtained from the Korea Institute of Oriental Medicine (Daejeon, Korea). SCWE (100 and $200 \mathrm{mg} / \mathrm{kg}$ ) was administered to mice by oral gavage $1 \mathrm{~h}$ before CS exposure for 7 days. The $200 \mathrm{mg} / \mathrm{kg}$ of SCWE is corresponding to $12 \mathrm{~g} / 60 \mathrm{~kg}(60 \mathrm{~g} / 60 \mathrm{~kg}$ as dried herbal amount) for human dose. As generally two formulas can be used as clinical daily dose, the dose used in this experiment is reasonable.

\section{Collection of Broncho-Alveolar Lavage Fluid (BALF)}

Forty-eight hours after the last intranasal LPS administration, the mice were sacrificed via an intraperitoneal injection of zoletil 50 (25 mg/kg; Virbac Korea. Co., Seoul, Korea), and a tracheostomy was performed. To obtain broncho-alveolar lavage fluid (BALF), ice-cold phosphate buffered saline (PBS; $0.7 \mathrm{~mL}$ ) was infused into the lung and withdrawn via the tracheal cannulation. This process was repeated once (total volume $1.4 \mathrm{~mL}$ ). To determine the differential cell counts, $100 \mu \mathrm{L}$ of BALF was centrifuged onto slides using Cytospin (Hanil Science Industrial, Seoul, Korea). The slides were dried, and the cells were fixed and stained using Diff-Quik $^{\circledR}$ staining reagent (B4132-1A; IMEB Inc., Deerfield, IL, United States) according to the manufacturer's instructions. The supernatant obtained from BALF was stored at $-70^{\circ} \mathrm{C}$ for biochemical analysis.

\section{Measurement of Proinflammatory Mediator in BALF}

The proinflammatory mediators in BALF were measured using enzyme-linked immunosorbent assay (ELISA) kits (TNF- $\alpha$; 558534, IL-6; 555240, IL-1 $\beta$; 559603, BD Science, San Diego, CA, United States) according to the manufacturer's protocols. The plates were incubated for $10 \mathrm{~min}$ in the dark, and the absorbance was measured at $450 \mathrm{~nm}$ in a microplate reader (Bio-Rad Laboratories, Hercules, CA, United States).

\section{Immunoblotting}

The lung tissue was homogenized (1/10 w/v) using a homogenizer in lysis buffer (tissue lysis/extraction reagent that contained a protease inhibitor cocktail). The cells were treated as described in the previous sections and then incubated in the presence of CSC $(20 \mu \mathrm{g} / \mathrm{mL})$ for $24 \mathrm{~h}$. The cells were collected by centrifugation, washed twice with PBS, and suspended using lysis buffer. The protein concentrations of 
the samples were determined using Bradford reagent (Bio$\mathrm{Rad})$. Equal amounts of total protein $(30 \mu \mathrm{g})$ were resolved by $10 \%$ sodium dodecyl sulfate (SDS)-polyacrylamide gel electrophoresis (PAGE) and transferred to nitrocellulose membranes. The membranes were incubated with blocking solution (5\% skim milk) followed by overnight incubation at $4^{\circ} \mathrm{C}$ with the appropriate primary antibody. The following primary antibodies and dilutions were used: anti- $\beta$-actin (1:2000 dilution; Cell Signaling, Beverly, MA, United States), anti$\mathrm{pI} \kappa \mathrm{B} \alpha$ (1:1000 dilution; Santa Cruz Biotechnology, Dallas, TX, United States), anti-I kappa B alpha (I $\mathrm{B} \alpha ; 1: 1000$ dilution; Santa Cruz), anti-pNF-кB (1:1000 dilution; Abcam, Cambridge, United Kingdom), anti-NF- $\kappa$ B (1:1000 dilution; Abcam), and anti-iNOS (1:1000 dilution; Santa Cruz). The blots were washed three times with Tris-buffered saline containing Tween 20 (TBST) and then incubated with a 1:10,000 dilution of horseradish peroxidase (HRP)-conjugated secondary antibody (Jackson Immuno Research, West Grove, PA, United States) for $30 \mathrm{~min}$ at room temperature. The blots were again washed three times with TBST and then developed using an enhanced chemiluminescence (ECL) kit (Thermo Fisher Scientific, Boston, MA, United States).

\section{Gelatin Zymography}

SDS-PAGE zymography was performed as described in Heussen and Dowdle (1980) to determine gelatinase activities. Briefly, zymogram gels consisting of $10 \%$ SDS-PAGE containing $1 \%$ gelatin were used as the MMP substrate. The gels were washed in $2.5 \%$ Triton $\mathrm{X}-100$ for $1 \mathrm{~h}$ to remove SDS and then incubated at $37^{\circ} \mathrm{C}$ for $16 \mathrm{~h}$ in developing buffer ( $1 \mathrm{M}$ Tris- $\mathrm{HCl}, \mathrm{pH} 7.5$ with $\mathrm{CaCl}_{2}$ ). Thereafter, gels were stained with $25 \%$ methanol/8\% acetic acid containing Coomassie Brilliant Blue. Gelatinase activity was visualized as white bands on a blue background that represented the areas of proteolysis.

\section{Lung Tissue Histopathology}

The lung tissue was fixed in $4 \%(\mathrm{v} / \mathrm{v})$ paraformaldehyde, embedded in paraffin, sectioned at $4 \mu \mathrm{m}$ thickness, and stained with hematoxylin and eosin (H\&E solution, Sigma-Aldrich) to estimate inflammation.

Immunohistochemical slides were deparaffinized, dehydrated, washed in PBS containing 0.05\% tween 20 (PBS-T), and incubated for $20 \mathrm{~min}$ at room temperature with goat serum to block non-specific staining. The slides were incubated for $2 \mathrm{~h}$ at room temperature with primary mouse anti-mouse MMP-9 antibody (diluted 1:100, Abcam). After incubation, they were washed three times, incubated for $1 \mathrm{~h}$ at room temperature with biotinylated secondary antibody, and then incubated with an avidin-biotin-peroxidase complex (Vector Laboratories, Burlingame, CA, United States) for $1 \mathrm{~h}$ at room temperature. Then, the slides were washed with PBS-T and incubated with diaminobenzidine (DAB, Abcam) for an additional $5 \mathrm{~min}$.

\section{Statistical Analysis}

The data are expressed as means \pm standard deviation (SD). Statistical significance was determined using an analysis of variance (ANOVA) followed by a multiple comparison test with Dunnet's adjustment. $P$-values $<0.05$ were considered significant.

\section{RESULTS}

\section{HPLC Analysis of SCWE Decoction}

For quality assessment of the SCWE decoction, HPLC analysis was conducted using gradient elution of two mobile systems. Nine marker ingredients were completely separated within $35 \mathrm{~min}$; the representative HPLC chromatogram is shown in Figure 1. For simultaneous analysis of the SCWE decoction, the detection wavelength of the eight marker compounds was set at $230 \mathrm{~nm}$ (albiflorin and paeoniflorin), $254 \mathrm{~nm}$ (glycyrrhizin and schizandrin), and $280 \mathrm{~nm}$ (liquiritin, coumarin, cinnamic acid, and cinnamaldehyde). The retention times of albiflorin, paeoniflorin, liquiritin, coumarin, cinnamic acid, cinnamaldehyde, glycyrrhizin, and schizandrin were detected at approximately $8.77,9.65,11.60,17.53,20.57,23.07,30.59$, and $31.44 \mathrm{~min}$, respectively. Using an established HPLC-PDA method, the amounts of albiflorin, paeoniflorin, liquiritin, coumarin, cinnamic acid, cinnamaldehyde, glycyrrhizin, and schizandrin in the freeze-dried SCWE decoction were found to be $2.19,11.48,3.87,0.19,0.31,0.24,0.69$, and $0.52 \mathrm{mg} / \mathrm{g}$, respectively.

\section{SCWE Reduces Inflammatory Cytokines in CSC-Stimulated NCl-H292 Cells}

In this study, the ability of non-toxic concentrations of SCWE $(1.25,2.5,5$, and $10 \mu \mathrm{g} / \mathrm{mL})$ to inhibit the mRNA expression of CSC-stimulated TNF- $\alpha$, IL- 6 , and IL- $1 \beta$. The expression levels of TNF- $\alpha$, IL- 6 and IL- $1 \beta$ increased in CSC-stimulated cells compared to non-stimulated cells (Figure 2). The levels of TNF- $\alpha$, IL- 6 and IL-1 $\beta$ decreased in SCWE-treated cells in a concentration-dependent manner compared to the CSCstimulated cells.

\section{SCWE Inhibits iNOS and Phosphorylation of NF-кB Expression in CSC-Stimulated NCl-H292 Cells}

The iNOS and phosphorylation of NF- $\mathrm{KB}$ expression increased CSC-stimulated cells compared to the nonstimulated cells. The iNOS and phosphorylation of NF-кB induced by CSC markedly decreased by SCWE treatment (Figure 3).

\section{SCWE Decreases the Number of Inflammatory Cells in BALF Induced by CS and LPS Exposure}

The number of inflammatory cells in BALF was increased in CS and LPS exposed mice compared with vehicle control mice. Specifically, CS and LPS exposure markedly increased the number of neutrophils in BALF compared to control. In SCWE treated mice, however, the number of neutrophils in BALF was 


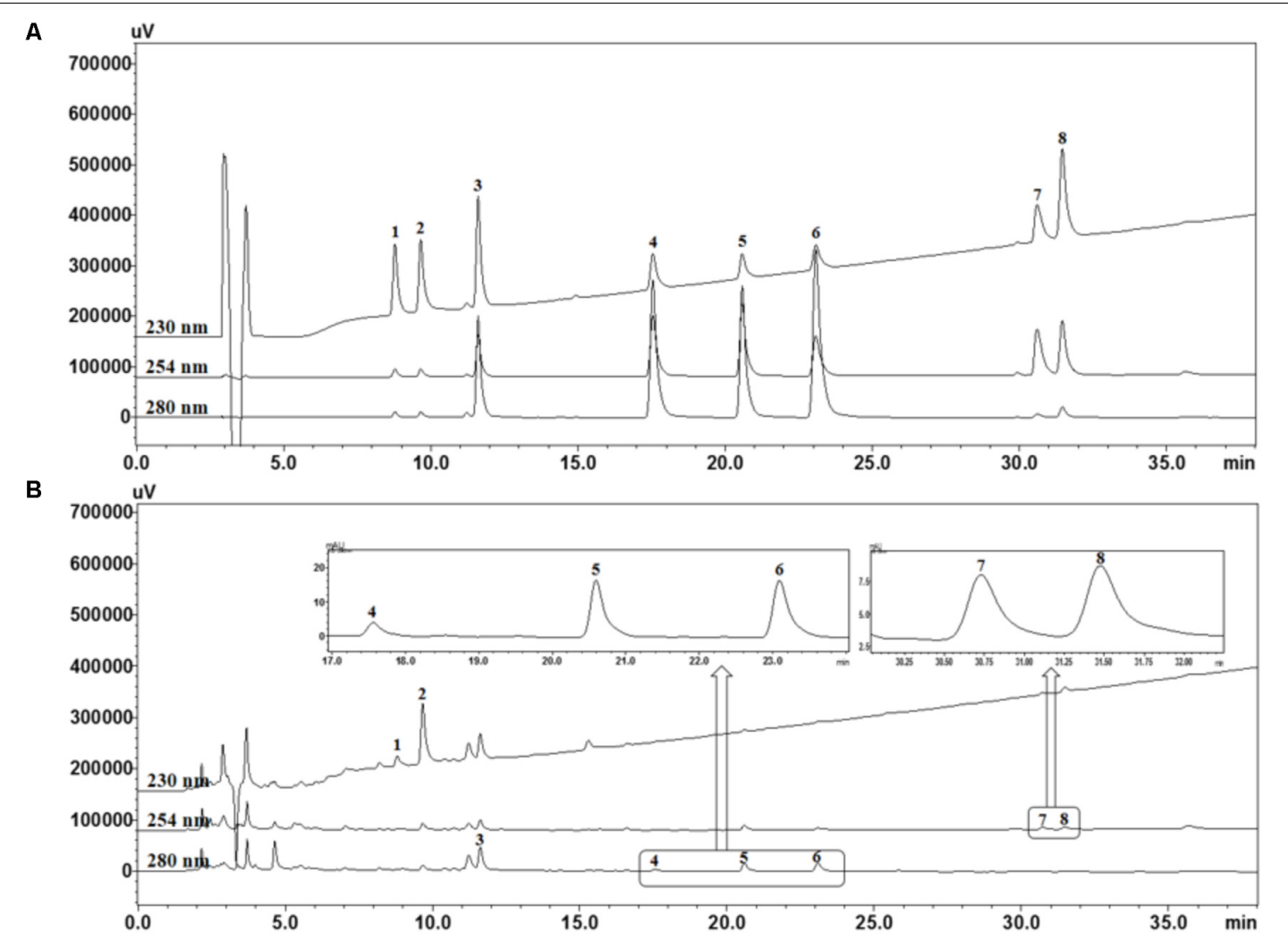

FIGURE 1 | High performance liquid chromatography (HPLC) chromatograms of standard mixtures (A) and So-Cheong-Ryoung-Tang water extract (SCWE) decoctions (B) at different detection wavelengths. Albiflorin (1), paeoniflorin (2), liquiritin (3), coumarin (4), cinnamic acid (5), cinnamaldehyde (6), glycyrrhizin (7), and schizandrin (8).
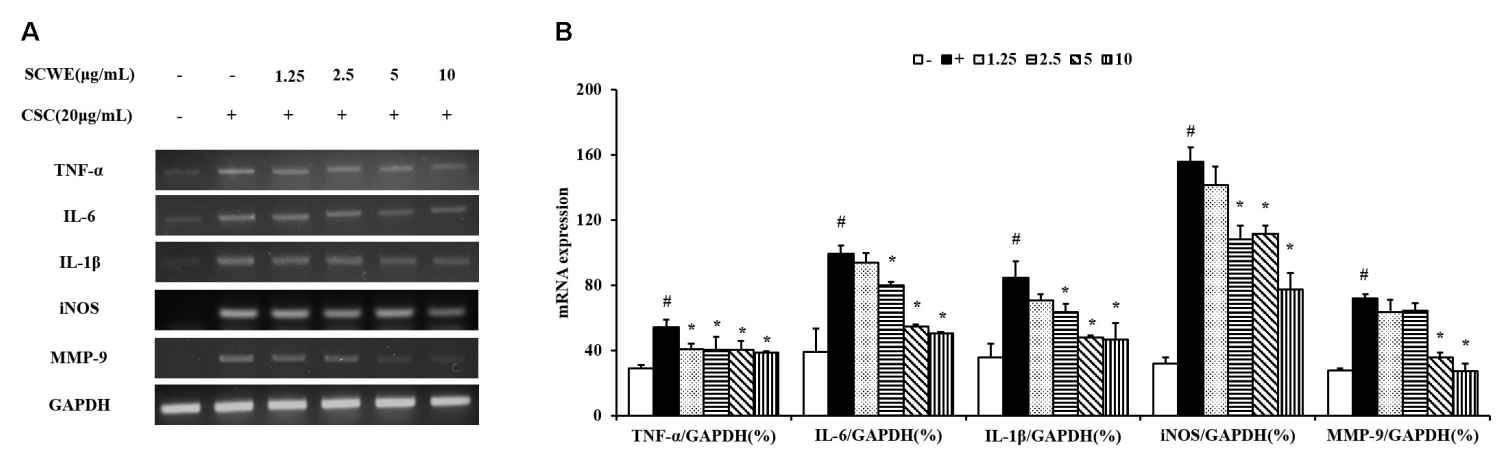

FIGURE 2 | SCWE reduces the expression of inflammatory cytokines in CSC-stimulated H292 cells. (A) CSC reduced the mRNA expression of TNF- $\alpha$, IL-6 and $\mathrm{IL}-1 \beta$. (B) Quantitative analysis for $\mathrm{mRNA}$ expression. The values are expressed as the means $\pm \mathrm{SD}$. \#Significantly different from the controls, $P<0.05$; *significantly different from the CSC-stimulated cells, $P<0.05$.

dose-dependently decreased compared with CS and LPS exposed mice (Figure 4).

\section{SCWE Reduces Proinflammatory Cytokines Induced by CS and LPS Exposure}

In CS and LPS exposed mice, the levels of TNF- $\alpha$ and IL- 6 in BALF were significantly increased compared to the vehicle control mice. Roflumilast treated mice significantly reduced TNF- $\alpha$ and IL-6 in CS and LPS exposed mice (Figure 5). In addition, SCWE treated mice dose dependently decreased TNF$\alpha$ and IL- 6 levels compared with CS and LPS exposed mice. The results of IL- $1 \beta$ in BALF were similar to those of TNF- $\alpha$ and IL- 6 . CS and LPS exposure mice significantly increased the level of IL$1 \beta$ in BALF compared with the vehicle control mice, and SCWE treated mice significantly decreased the level of IL- $1 \beta$ in BALF in CS and LPS exposed mice. 

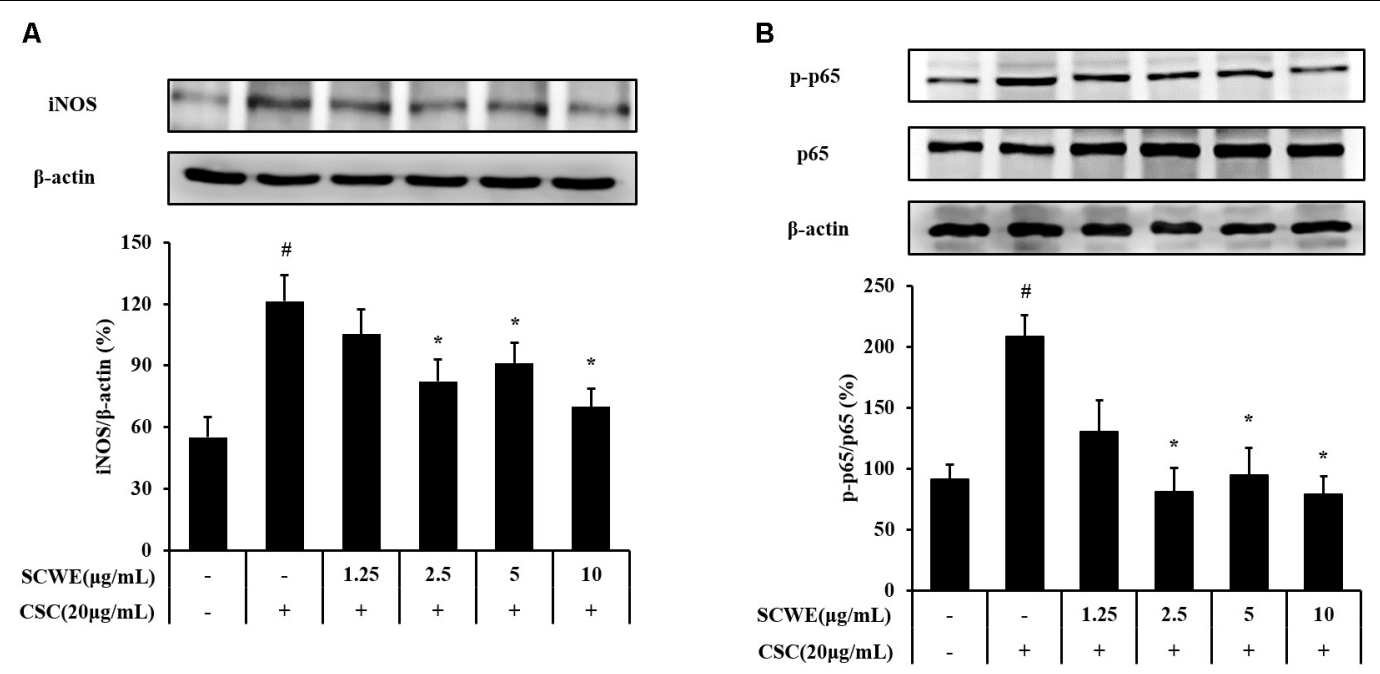

FIGURE 3 | SCWE inhibited the iNOS and phosphorylation of NF-кB expression in CSC-stimulated H292 cells. (A) SCWE inhibited the iNOS expression and

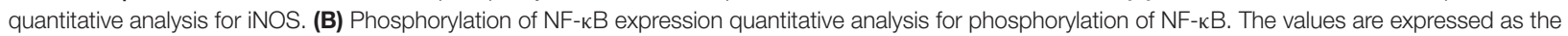
means \pm SD. \#Significantly different from the controls, $P<0.05$; *significantly different from the CSC-stimulated cells, $P<0.05$.

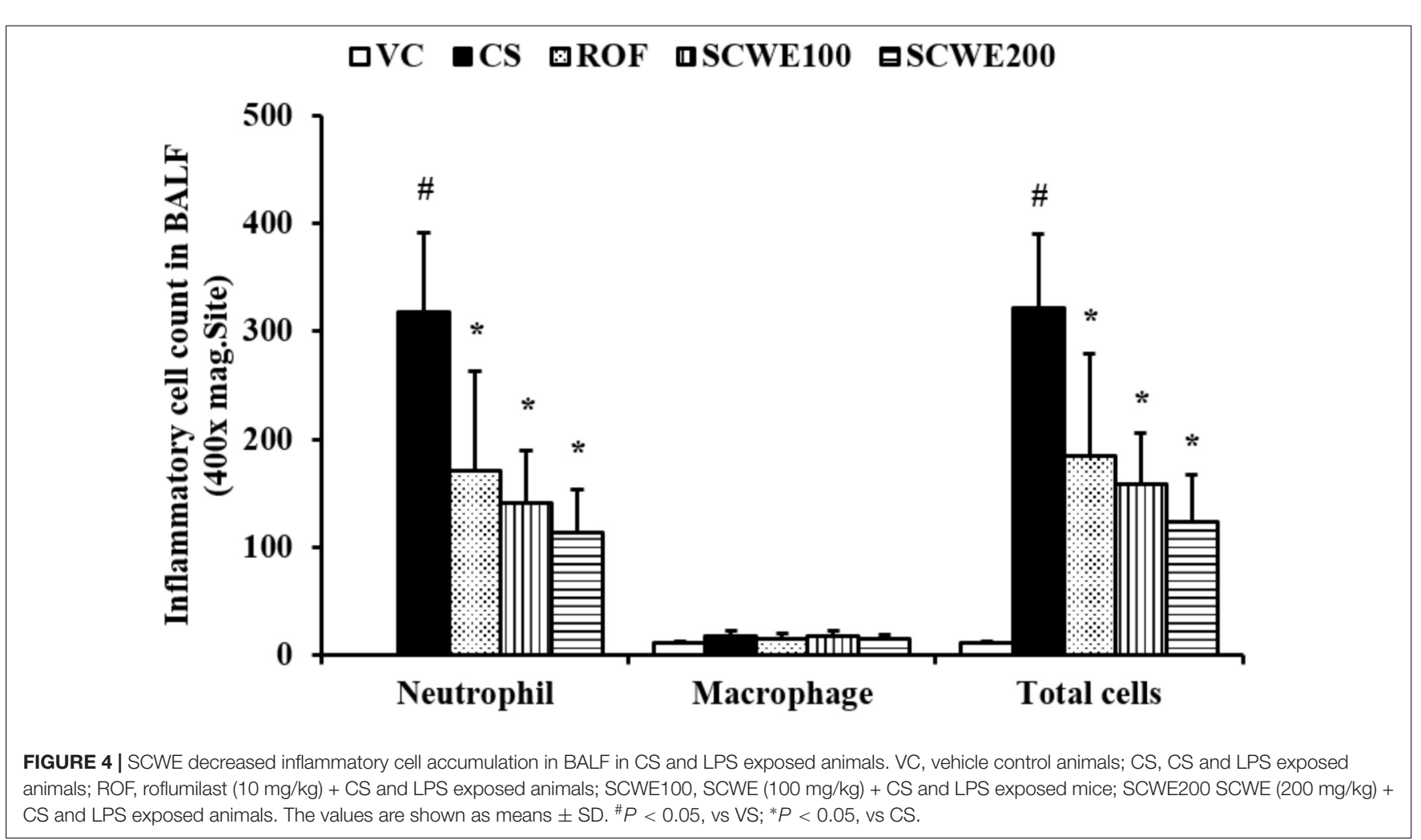

\section{SCWE Decreases the Expression of}

Proinflammatory Mediators in Lung Tissue Induced by CS and LPS Exposure

Inducible nitric oxide synthase expression was increased in the lung tissue of CS and LPS exposed mice compared to vehicle control mice. Roflumilast treated mice markedly decreased iNOS expression in the lung tissue compared with CS and LPS exposed mice. In addition, SCWE treated mice dose-dependently decreased iNOS expression in lung tissue compared with CS and LPS exposed mice (Figures 6A,B).

In comparison to vehicle control mice, the phosphorylation of IкB $\alpha$ was significantly increased in CS and LPS exposed mice. SCWE treated mice markedly and dose-dependently decreased phosphorylation of I $\mathrm{I} B \alpha$ in CS and LPS exposed mice. Similarly, NF- $\mathrm{KB}$ phosphorylation in lung tissue was markedly increased 

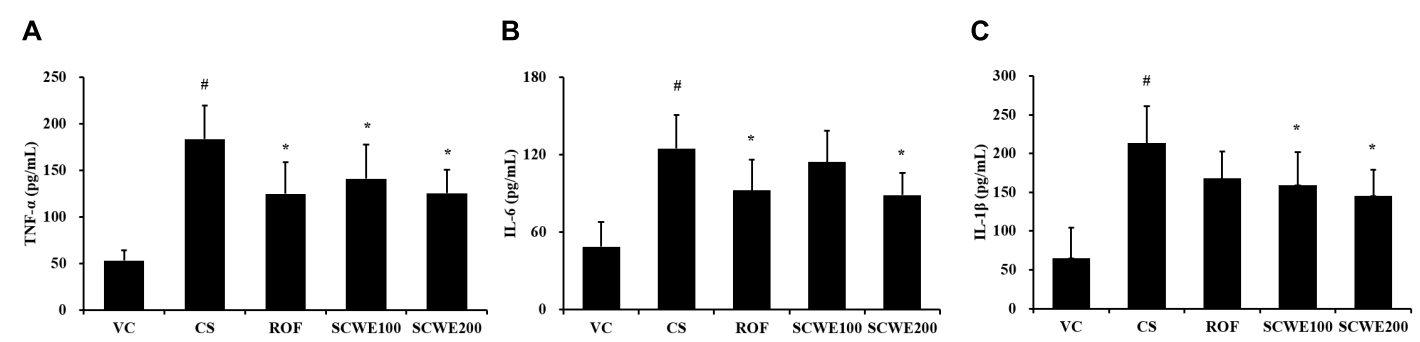

FIGURE 5 | SCWE declined the inflammatory cytokines production caused by CS and LPS exposure. (A) Tumor necrosis factor alpha (TNF- $\alpha$ ), (B) interleukin (IL)-6, and (C) IL-1 $\beta$. VC, vehicle control animals; CS, CS and LPS exposed animals; ROF, roflumilast (10 mg/kg) + CS and LPS exposed animals; SCWE100, SCWE $(100 \mathrm{mg} / \mathrm{kg})+\mathrm{CS}$ and LPS exposed animals; SCWE200, SCWE $(200 \mathrm{mg} / \mathrm{kg})+\mathrm{CS}$ and LPS exposed animals. The values are shown as means \pm SD. \# $P<0.05$, vs VS; ${ }^{*} P<0.05$, vs CS.

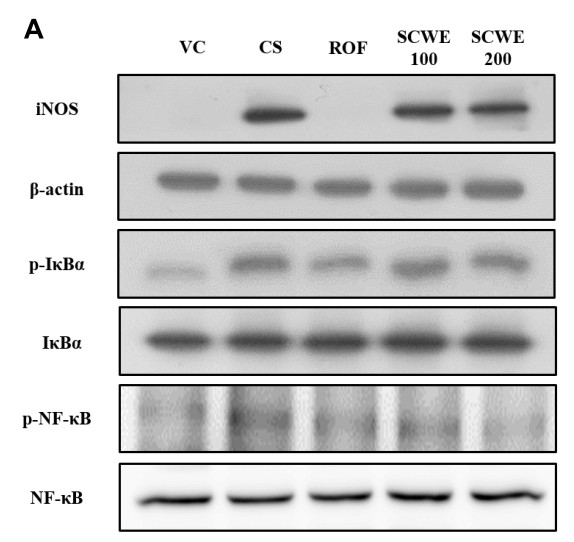

B

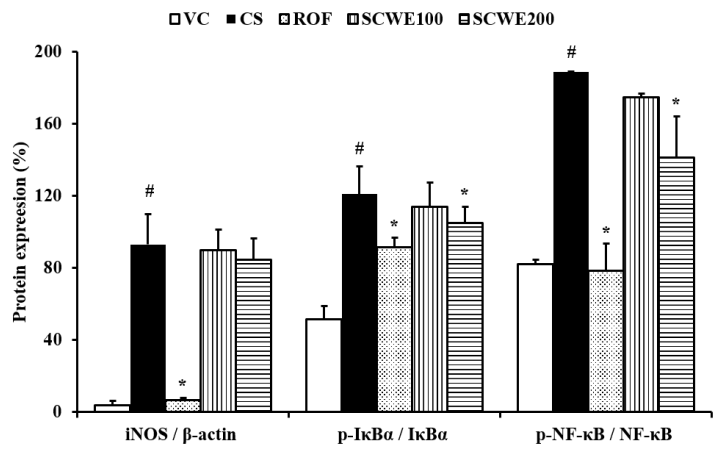

FIGURE 6 | SCWE inhibited inflammatory mediators expression in lung tissue stimulated by CS and LPS exposure. (A) Representative figure of protein expression. (B) Quantitative analysis for protein expression. VC, vehicle control animals; CS, CS and LPS exposed animals; ROF, roflumilast (10 mg/kg) + CS and LPS exposed animals; SCWE100, SCWE (100 mg/kg) + CS and LPS exposed animals; SCWE200, SCWE $(200 \mathrm{mg} / \mathrm{kg})+$ CS and LPS exposed animals. The values are shown as means \pm SD. ${ }^{\#} P<0.05$, vs VS; ${ }^{*} P<0.05$, vs CS.

in CS and LPS exposed mice compared to vehicle control mice. SCWE treated mice significantly decreased in a dose-dependent manner the phosphorylation of NF- $\mathrm{B}$ in CS and LPS exposed mice (Figures 6A,B).

\section{SCWE Attenuates Inflammatory Responses in Lung Tissue Induced by CS and LPS Exposure}

Cigarette smoke and LPS exposed mice exhibited extensive inflammatory cell infiltration into the lung tissue (Figure 7). Inflammatory cells mainly accumulating in peribronchial and alveolar lesions. In contrast, roflumilast treated mice decreased inflammatory cell infiltration into lung tissue induced by CS and LPS exposure. Similarly, inflammatory cell infiltration was significantly reduced in a dose-dependent manner in SCWE treated mice compared with CS and LPS exposed mice.

\section{SCWE Reduces the Expression and Activity of MMP-9 in Lung Tissue Induced by CS and LPS Exposure}

MMP-9 expression in lung tissue was markedly increased in CS and LPS exposed mice compared to vehicle control mice
(Figure 8A). SCWE treated mice, however, reduced this increased expression of MMP-9 in lung tissue induced by CS and LPS exposure. In zymographs, CS and LPS exposed mice showed a marked increase in MMP-9 activity compared with the vehicle control mice, whereas SCWE treated mice exhibited a marked and dose-dependent reduction in MMP-9 activity compared with CS and LPS exposed mice (Figure 8B).

\section{DISCUSSION}

In this study, we evaluated the protective effects of SCWE on airway inflammation induced by CS and LPS exposure. SCWE significantly reduced the elevation of inflammatory cell count and proinflammatory cytokines in BALF induced by CS and LPS exposure. SCWE effectively decreased the enhanced expression of iNOS and phosphorylation of I $\mathrm{B} \alpha$ and NF- $\kappa \mathrm{B}$ induced by CS and LPS exposure in lung tissue, which were accompanied by reductions in the inflammatory responses and MMP-9 expression in lung tissue.

Cigarette smoke is a major risk factor for the development and aggravation of COPD characterized by airflow limitation. CS leads to persistent airway inflammation, resulting in irreversible 


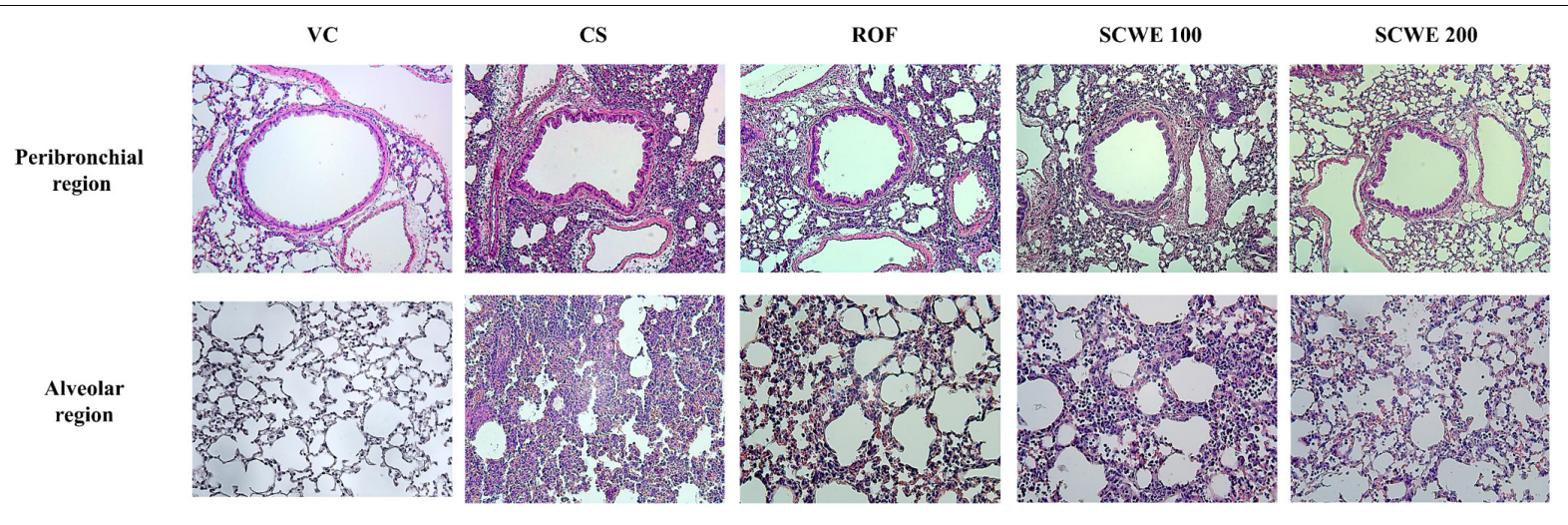

FIGURE 7 | SCWE decreased inflammatory cell infiltration caused by CS and LPS exposure. VC, vehicle control animals; CS, CS and LPS exposed animals; ROF, roflumilast $(10 \mathrm{mg} / \mathrm{kg})+$ CS and LPS exposed animals; SCWE100, SCWE (100 mg/kg) + CS and LPS exposed animals; SCWE200, SCWE (200 mg/kg) + CS and LPS exposed animals.

A

VC

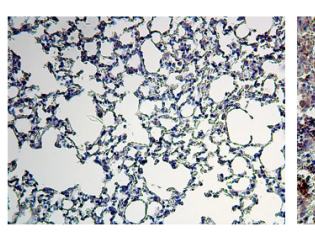

SCWE 100

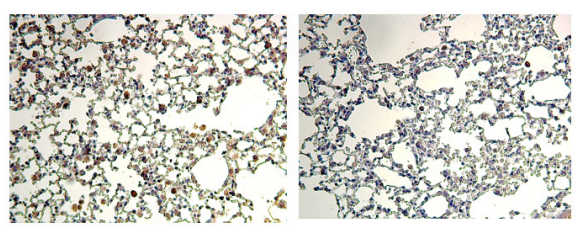

B

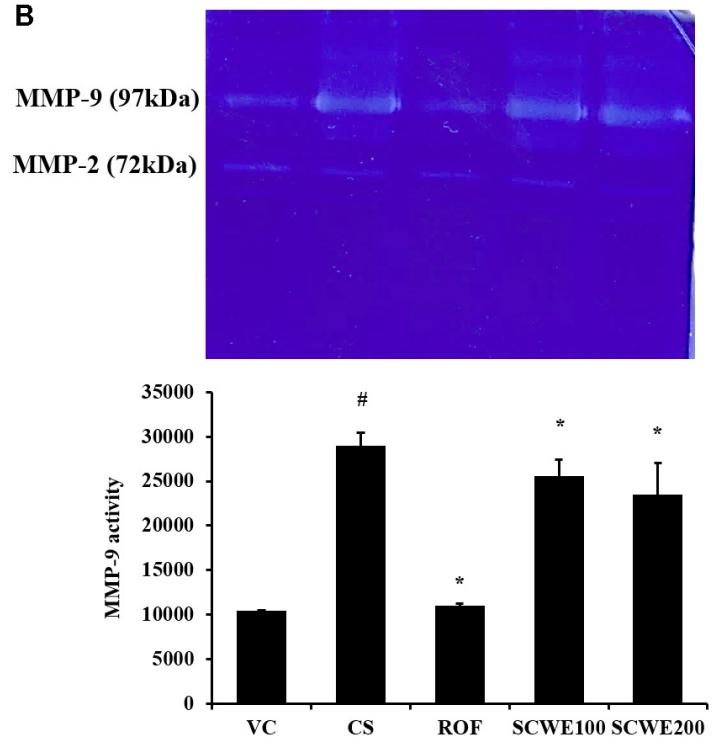

FIGURE 8 | SCWE reduced the expression and activity of MMP-9 stimulated by CS and LPS exposure. (A) Representative figure of MMP-9 expression. (B) MMP-9 activity. VC, vehicle control animals; CS, CS and LPS exposed animals; ROF, roflumilast (10 mg/kg) + CS and LPS exposed animals; SCWE100, SCWE (100 mg/kg) + CS and LPS exposed animals; SCWE200, SCWE $(200 \mathrm{mg} / \mathrm{kg})+\mathrm{CS}$ and LPS exposed animals. The values are shown as means \pm SD. ${ }^{\#} P<0.05$, vs VS; $* P<0.05$, vs CS.

structural changes in lung tissues (Lee et al., 2016). CS was shown to provoke an influx of inflammatory cells, such as neutrophils and macrophages, into the airway (Lee K.H. et al., 2015). Of the inflammatory cells, neutrophils have been associated with the production of various mediators, including cytokines, chemokines, and proteases (Gernez et al., 2010). These responses lead to aggravated airway inflammation and destroyed normal alveolar structure. In this study, SCWE significantly reduced inflammatory cell counts in a dose-dependent manner in CS and LPS exposed mice, and this reduction was accompanied by a reduction in the levels of TNF- $\alpha$, IL- 6 , and IL- $1 \beta$ in BALF and CSC-stimulated H292 cells. In addition, SCWE effectively attenuated airway inflammation induced by CS and LPS exposure. Taken together, these results suggest that SCWE may suppress airway inflammation induced by CS and LPS exposure.

The protease MMP-9 has an important role in various physiological and pathological processes (Hernández-Montoya et al., 2015). In development of COPD, MMP-9 was shown to be activated by various stimuli, such as CS, oxidative stress, and other airway proteases in the airway, and was involved in the destruction of normal alveolar structure, production of proinflammatory mediators, and inflammatory cell migration (Cane et al., 2016; Grzela et al., 2016). In a clinical trial, patients with COPD had increased expression of MMP-9 in their sputum and lavage (Mercer et al., 2005). MMP-9 expression is closely associated with activation of $N F-\kappa B$, which is involved in the process of airway inflammation (Pang et al., 2015). 
$\mathrm{NF}-\kappa \mathrm{B}$ is also involved in the regulation of proinflammatory and immune regulatory pathways (Jones et al., 2016). Normally, $\mathrm{NF}-\kappa \mathrm{B}$ is bound to I $\mathrm{B} \alpha$ in the cytosol in an inactive form, and upon degradation of $\mathrm{I} \kappa \mathrm{B} \alpha$ by selective ubiquitination, NF$\kappa B$ is activated (Kwon et al., 2016). Activated NF- $\kappa$ B finally produces MMP-9 and inflammatory cytokines (Zhang et al., 2016). Recently, a study showed that the expression of MMP9 along with NF- $\mathrm{B}$ was increased in CS extract stimulated peripheral blood mononuclear cells (PBMCs) and murine alveolar macrophage cells and in a CS-induced emphysema rat model (Hou et al., 2015; Li et al., 2015). In addition, $\mathrm{NF}-\kappa \mathrm{B}$ activation was demonstrated to be required for the increase in iNOS expression involved in the pathogenesis of lung injury (Li et al., 2014). iNOS expression is a key molecule that protects lungs from inflammation (Roh et al., 2010). iNOS expression is upregulated by various proinflammatory signals, resulting in elevations in NO concentrations (Hesslinger et al., 2009). NO production, an active player in the respiratory system and disease, is considered a potential mediator of airway responsiveness (Jiang et al., 2006). In this study, CS and LPS exposed mice exhibited marked increases in MMP-9 and iNOS expression in lung tissue, which were accompanied by elevations in the phosphorylation of NF$\kappa \mathrm{B}$ and $\mathrm{I} \kappa \mathrm{B} \alpha$. Importantly, SCWE treated mice significantly decreased the expression of MMP-9 and iNOS with reduction the phosphorylation of NF- $\kappa \mathrm{B}$ and $\mathrm{I} \kappa \mathrm{B} \alpha$ in lung tissue in CS and LPS exposed mice. Also, SCWE reduced expression of iNOS and phosphorylation of NF- $\kappa \mathrm{B}$ in CSC-stimulated cells. These results indicate that the protective effect of SCWE is closely associated with the suppression of the NF- $\kappa \mathrm{B}$ pathway, which resulted in the reduction in expression of MMP-9 and iNOS.

The traditional herbal formula So-Cheong-Ryoung-Tang is a mixture of eight herbal preparations (Supplementary Table S1) and is used to treat pulmonary diseases such as bronchitis, asthma, and cough (Ko et al., 2004; Lee M.Y. et al., 2015). Pharmacological studies have demonstrated that So-Cheong-Ryoung-Tang possesses protective effects against airway inflammation, nasal allergy, pulmonary fibrosis, and asthma (Kao et al., 2000; Das et al., 2009; Yang et al., 2010). In a recent study, So-Cheong-Ryoung-Tang effectively suppressed the production of MMP-9 in human bronchial epithelium cell line BEAS-2B stimulated with TNF- $\alpha$ and IL-4. In a murine model of ovalbumin-induced allergic asthma, SCWE reduced the levels of IL-17 and granulocyte/macrophage colony-stimulating factor (GM-CSF) with reduction in the level of IL-4, which eventually decreased inflammatory cell infiltration and airway remodeling resulting clinical signs of allergic asthma (Kim et al., 2014). This anti-inflammatory properties evidenced by previous studies were strongly consistent with our results. In addition, anti-inflammatory properties of SCWE are associated with not only Iк $\mathrm{B} \alpha / \mathrm{NF}-\kappa \mathrm{B}$ signaling but other signaling such as MAPKs. In particular, MAPKs play an important player in the inflammatory responses such as the production of proinflammatory cytokines and chemokines (Turner et al., 2014). However, SCWE has been reported to increase the MAPKs expression in the literature to date
(Hwang et al., 2013; Yim et al., 2015). SCWE induces apoptosis via enhancement of MAPK signaling pathway in cancer cells and affects GI motility by the modulation of pacemaker activity through MAPKs in interstitial cells of Cajal. But, previous studies are performed under specific condition including cancer cells without stimulation materials such as LPS, CSC, etc., and ex vivo experiments. In addition, anticancer effect of SCWE was induced by the alteration of its active components by bacterial fermentation (Yim et al., 2015). There is no precise description of what reaction will occur under disease conditions such as asthma and COPD until now. Therefore, further research clearly needed to determine the effects of SCWE on MAPKs expression under airway inflammation.

\section{CONCLUSION}

The present study showed that SCWE suppresses the production of inflammatory mediators and MMP-9 activity in CS and LPS induced mice and CSC-stimulated H292 cells. These effects may be associated with the inhibition of CS-mediated I $\mathrm{B} \alpha / \mathrm{NF}-\kappa \mathrm{B}$ signaling. Therefore, SCWE is a potential treatment for lung disease, such as CS induced lung inflammation.

\section{ETHICS STATEMENT}

All procedures were performed in compliance with the Guide for the Care and Use of Laboratory Animals of the National Institutes of Health and Korean National Laws for Animal Welfare.

\section{AUTHOR CONTRIBUTIONS}

N-RS and I-SS conceived and designed the experiments. $\mathrm{N}-\mathrm{RS}$ and J-WK performed the experiments. J-SK, CK, and Y-KC analyzed the data. J-SK, J-CK, and C-SS contributed reagents, materials, and analysis tool. N-RS wrote the manuscript.

\section{FUNDING}

This work was supported by the National Research Foundation of Korea (NRF) grant funded by the Ministry of Science, ICT, and Future Planning (Grant No. NRF-2016R1C1B2008818) and Maximize utilization of knowledge about herbal resource (Grant No. K18404) funded by the Korea Institute of Oriental Medicine.

\section{SUPPLEMENTARY MATERIAL}

The Supplementary Material for this article can be found online at: https://www.frontiersin.org/articles/10.3389/fphar.2018. 01064/full\#supplementary-material 


\section{REFERENCES}

Cane, J. L., Mallia-Millanes, B., Forrester, D. L., Knox, A. J., Bolton, C. E., and Johnson, S. R. (2016). Matrix metalloproteinases -8 and -9 in the airways, blood and urine during exacerbations of copd. COPD 13, 26-34. doi: 10.3109/ 15412555.2015.1043522

Das, A. K., Mizuguchi, H., Kodama, M., Dev, S., Umehara, H., Kitamura, Y., et al. (2009). Sho-seiryu-to suppresses histamine signaling at the transcriptional level in TDI-sensitized nasal allergy model rats. Allergol. Int. 58, 81-88. doi: 10.2332/allergolint.O-07-526

Dong, R., Xie, L., Zhao, K., Zhang, Q., Zhou, M., and He, P. (2015). Cigarette smoke-induced lung inflammation in COPD mediated via LTB4/BLT1/SOCS1 pathway. Int. J. Chron. Obstruct. Pulmon. Dis. 11, 31-41. doi: 10.2147/COPD. S96412

Ge, L. T., Liu, X. X., Shen, H. J., Jia, Y. L., Cong, X. W., Sun, Y., et al. (2016). Inhalation of ambroxol inhibits cigarette smoke-induced acute lung injury in a mouse model by inhibiting the Erk pathway. Int. Immunopharmacol. 33, 90-98. doi: 10.1016/j.intimp.2016.02.004

Gernez, Y., Tirouvanziam, R., and Chanez, P. (2010). Neutrophils in chronic inflammatory airway diseases: can we target them and how? Eur. Respir. J. 35, 467-469. doi: 10.1183/09031936.00186109

Grzela, K., Litwiniuk, M., Zagorska, W., and Grzela, T. (2016). Airway remodeling in chronic obstructive pulmonary disease and asthma: the role of matrix metalloproteinase-9. Arch. Immunol. Ther. Exp. 64, 47-55. doi: 10.1007/ s00005-015-0345-y

Hou, G., Yin, Y., Han, D., Wang, Q. Y., and Kang, J. (2015). Rosiglitazone attenuates the metalloprotease/anti-metalloprotease imbalance in emphysema induced by cigarette smoke: involvement of extracellular signal-regulated kinase and NF-кB signaling. Int. J. Chron. Obstruct. Pulmon. Dis. 10, 715-724. doi: 10.2147/COPD.S77514

Hernández-Montoya, J., Pérez-Ramos, J., Montaño, M., Ramírez-Venegas, A., Sansores, R. H., Pérez-Rubio, G., et al. (2015). Genetic polymorphisms of matrix metalloproteinases and protein levels in chronic obstructive pulmonary disease in a Mexican population. Biomark. Med. 9, 979-788. doi: 10.2217/bmm. 15.75

Hesslingere, C., Lehner, M. D., Strub, A., Boer, R., Ulrich, W. R., Kuelzer, R., et al. (2008). The highly selective iNOS inhibitor BYK402750 exerts potent anti-inflammatory effects in a mouse model of cigarette smokeinduced inflammation. Nitric Oxide 19:35. doi: 10.1016/j.niox.2008. 06.067

Hesslinger, C., Strub, Z., Boer, R., Ulrich, W. R., Lehner, M. D., and Braun, C. (2009). Inhibition of inducible nitric oxide synthase in respiratory diseases. Biochem. Soc. Trans. 37, 886-891. doi: 10.1042/BST037 0886

Heussen, C., and Dowdle, D. B. (1980). Electrophoretic analysis of plasminogen activators in polyacrylamide gels containing sodium dodecyl sulfate and copolymerized substrates. Anal. Biochem. 102, 196-202. doi: 10.1016/00032697(80)90338-3

Hwang, M. W., Lee, H. J., Song, H. J., and Kim, B. J. (2013). Involvement of MAPKs and PLC pathways in modulation of pacemaking activity by So-Cheong-Ryong-Tang in interstitial cells of Cajal from murine small intestine. ScientificWorldJournal 2013:536350. doi: 10.1155/2013/ 536350

Jiang, H., Qu, J., He, L., Pan, J., Chen, X., Li, L., et al. (2006). Airway hyperresponsiveness induced by repetitive intraperitoneal injection of lipopolysacharide and the involvement of inflammation and nitric oxide in guinea pigs. Inflamm. Res. 55, 286-292. doi: 10.1007/s00011-0060085-x

Jiang, W. T., Liu, X. S., Xu, Y. J., Ni, W., and Chen, S. X. (2015). expression of nitric oxide synthase isoenzyme in lung tissue of smokers with and without chronic obstructive pulmonary disease. Chin. Med. J. 128, 1584-1589. doi: 10.4103/0366-6999.158309

Jones, J. T., Qian, X., van der Velden, J. L., Chia, S. B., McMillan, D. H., Flemer, S., et al. (2016). Glutathione S-transferase pi modulates NF- $\kappa B$ activation and pro-inflammatory responses in lung epithelial cells. Redox Biol. 26, 375-382. doi: 10.1016/j.redox.2016.03.005

Jung, K. H., Beak, H., Park, S., Jung, J., Park, S., Kim, J., et al. (2016a). The therapeutic effects of tuberostemonine against cigarette smoke-induced acute lung inflammation in mice. Eur. J. Pharmacol. 774, 80-86. doi: 10.1016/j.ejphar. 2016.02.006

Jung, K. H., Kil, Y. S., Jung, J., Park, S., Shin, D., Lee, K., et al. (2016b). Tuberostemonine N, an active compound isolated from Stemona tuberosa, suppresses cigarette smoke-induced sub-acute lung inflammation in mice. Phytomedicine 23, 79-86. doi: 10.1016/j.phymed.2015.11.015

Kao, S. T., Wang, S. D., Wang, J. Y., Yu, C. K., and Lei, H. Y. (2000). The effect of Chinese herbal medicine, xiao-qing-long-tang (XQLT), on allergen-induced bronchial inflammation in mite-sensitized mice. Allergy 55, 1127-1133. doi: 10.1034/j.1398-9995.2000.00728.x

Kim, H. W., Lim, C. Y., Kim, B. Y., and Cho, S. I. (2014). So-Cheong-Ryong-Tang, a herbal medicine, modulates inflammatory cell infiltration and prevents airway remodeling via regulation of interleukin-17 and GM-CSF in allergic asthma in mice. Pharmacogn. Mag. 10(Suppl. 3), S506-S511. doi: 10.4103/0973-1296. 139784

Ko, E., Rho, S., Seo, Y. H., Cho, C., Lee, Y., Min, B. I., et al. (2004). Traditional Korean medicine (SCRT) modulate Th1/Th2 specific cytokine production in mice CD4+ T cell. J. Ethnopharmacol. 92, 121-128. doi: 10.1016/j.jep.2004 02.008

Kwon, S. H., Ma, S. X., Hwang, J. Y., Ko, Y. H., Seo, J. Y., Lee, B. R., et al. (2016). The anti-inflammatory activity of Eucommia ulmoides oliv. Bark. Involves NF- $\kappa \mathrm{B}$ suppression and Nrf2-dependent HO-1 induction in BV-2 microglial cells. Biomol. Ther. 24, 268-282. doi: 10.4062/biomolther. 2015.150

Ku, J. M., Hong, S. H., Kim, S. R., Choi, H. S., Seo, H. S., Jang, B. H., et al. (2016). Anti-allergic effects of So-Cheong-Ryong-Tang in ovalbumin-induced allergic rhinitis model. Eur. Arch. Otorhinolaryngol. 273, 123-131. doi: 10.1007/s00405015-3515-y

Lee, E., Yun, N., Jang, Y. P., and Kim, J. (2013). Lilium lancifolium thunb. Extract attenuates pulmonary inflammation and air space enlargement in a cigarette smoke-exposed mouse model. J. Ethnopharmacol. 149, 148-156. doi: 10.1016/j. jep.2013.06.014

Lee, H., Park, J. R., Kim, E. J., Kim, W. J., Hong, S. H., Park, S. M., et al. (2016). Cigarette smoke-mediated oxidative stress induces apoptosis via the MAPKs/STAT1 pathway in mouse lung fibroblasts. Toxicol. Lett. 240, 140-148. doi: 10.1016/j.toxlet.2015.10.030

Lee, K. H., Lee, C. H., Jeong, J., Jang, A. H., and Yoo, C. G. (2015). Neutrophil elastase differentially regulates interleukin 8 (il-8) and vascular endothelial growth factor (VEGF) production by cigarette smoke extract. J. Biol. Chem. 290, 28438-28445. doi: 10.1074/jbc.M115.663567

Lee, M. Y., Seo, C. S., Kim, J. Y., and Shin, H. K. (2015). Evaluation of a water extract of So-Cheong-Ryong-Tang for acute toxicity and genotoxicity using in vitro and in vivo tests. BMC Complement. Altern. Med. 15:235. doi: 10.1186/s12906-0150737-x

Li, L., Wang, Y., Gao, W., Yuan, C., Zhang, S., Zhou, H., et al. (2015). Klotho reduction in alveolar macrophages contributes to cigarette smoke extractinduced inflammation in chronic obstructive pulmonary disease. J. Biol. Chem. 290, 27890-27900. doi: 10.1074/jbc.M115.655431

Li, X., Liu, Z., and Jin, H. (2014). Agmatine protects against zymosaninduced acute lung injury in mice by inhibiting NF-кB-mediated inflammatory response. Biomed. Res. Int. 2014:583736. doi: 10.1155/2014/ 583736

Mercer, P. F., Shute, J. K., and Bhowmik, A. (2005). MMP-9, TIMP-1 and inflammatory cells in sputum from COPD patients during exacerbation. Respir. Res. 6:151. doi: 10.1186/1465-9921-6-151

Pang, M., Wang, H., Bai, J.Z., Cao, D., Jiang, Y., Zhang, C., et al. (2015). Recombinant rat CC16 protein inhibits LPS-induced MMP-9 expression via NF-кB pathway in rat tracheal epithelial cells. Exp. Biol. Med. 240, 1266-1278. doi: $10.1177 / 1535370215570202$

Park, C., Hong, S. H., Kim, G. Y., et al. (2015). So-Cheong-Ryong-Tang induces apoptosis through activation of the intrinsic and extrinsic apoptosis pathways, and inhibition of the PI3K/Akt signaling pathway in non-small-cell lung cancer A549 cells. BMC Complement. Altern. Med. 15:113. doi: 10.1186/s12906-0150639-y

Roh, G. S., Yi, C. O., and Cho, Y. J. (2010). Anti-inflammatory effects of celecoxib in rat lungs with smoke-induced emphysema. Am. J. Physiol. Lung Cell Mol. Physiol. 299, L184-L191. doi: 10.1152/ajplung.00303. 2009 
Turner, M. D., Nedjai, B., Hurst, T., and Pennington, D. J. (2014). Cytokines and chemokines: at the crossroads of cell signalling and inflammatory disease. Biochim. Biophys. Acta. 1843, 2563-2582. doi: 10.1016/j.bbamcr.2014. 05.014

Wang, S. D., Lin, L. J., Chen, C. L., Lee, S. C., Lin, C. C., Wang, J. Y., et al. (2012). Xiao-Qing-Long-Tang attenuates allergic airway inflammation and remodeling in repetitive Dermatogoides pteronyssinus challenged chronic asthmatic mice model. J. Ethnopharmacol. 142, 531-538. doi: 10.1016/j.jep.2012.05.033

Yang, C. Q., Ishitsuka, Y., Moriuchi, H., Golbidi, S., Jin, Z. J., Irikura, M., et al. (2009). Protection afforded by a herbal medicine, Sho-seiryu-to (TJ-19), against oleic acid-induced acute lung injury in guinea-pigs. J. Pharm. Pharmacol. 61, 625-632. doi: 10.1211/jpp/61.07.0012

Yang, C. Q., Sun, P. Y., Ding, D. Z., Moriuchi, H., Ishitsuka, Y., Irikura, M., et al. (2010). The ethical Kampo formulation Sho-seiryu-to (TJ-19) prevents bleomycin-induced pulmonary fibrosis in rats. Biol. Pharm. Bull. 33, 1438-1442. doi: 10.1248/bpb.33.1438

Yim, M. H., Kim, A., Jung, Y. P., Kim, T., Ma, C. J., and Ma, J. Y. (2015). Fermented So-Cheong-Ryong-Tang (FCY) induces apoptosis via the activation of caspases and the regulation of MAPK signaling pathways in cancer cells. BMC Complement. Alter. Med. 15:336. doi: 10.1186/s12906-0150821-2

Zhang, X., Sun, C. Y., Zhang, Y. B., Guo, H. Z., Feng, X. X., Peng, S. Z., et al. (2016). Kegan Liyan oral liquid ameliorates lipopolysaccharide-induced acute lung injury through inhibition of TLR4-mediated NF-кB signaling pathway and MMP-9 expression. J. Ethnopharmacol. 186, 91-102. doi: 10.1016/j.jep.2016. 03.057

Conflict of Interest Statement: The authors declare that the research was conducted in the absence of any commercial or financial relationships that could be construed as a potential conflict of interest.

Copyright (c) 2018 Shin, Kim, Seo, Ko, Cho, Kim, Kim and Shin. This is an open-access article distributed under the terms of the Creative Commons Attribution License (CC BY). The use, distribution or reproduction in other forums is permitted, provided the original author(s) and the copyright owner(s) are credited and that the original publication in this journal is cited, in accordance with accepted academic practice. No use, distribution or reproduction is permitted which does not comply with these terms. 